\title{
Closed-loop effects in cardiovascular clinical decision support
}

\author{
Dirk Husmeier ${ }^{1}$ and L. Mihaela Paun \\ ${ }^{1}$ School of Mathematics and Statistics, University of Glasgow, \\ Glasgow G12 8SQ, UK \\ dirk.husmeier@glasgow.ac.uk; 1.paun.1@ research.gla.ac.uk
}

\begin{abstract}
We have recently seen impressive methodological developments in quantitative cardiovascular physiology and pathophysiology, with novel mathematical models for the mechanical and electrophysiological processes of the heart, and fluid dynamical models to describe the pressure and flow distribution in the blood vessel network. This allows us to gain deeper insight into the state of a variety of serious cardiovascular diseases. The majority of recent research studies have focused on the forward problem: developing flexible mathematical models and robust numerical simulation procedures to match characteristics of physiological target data, and the inverse problem: inferring model parameters from cardiac physiological data with reliable uncertainty quantification. However, when connecting mathematical model predictions and statistical inference to the clinical decision process, new challenges arise. This paper briefly discusses the complications that potentially result from closed-loop effects, and the model extensions that are required to reduce the ensuing bias.
\end{abstract}

Keywords: Physiological modelling, statistical inference, clinical decision support, closed-loop effects, prediction bias.

\section{Introduction and illustration}

Consider a random variable $X \in \mathbb{R}$ that represents the value of a clinical disease indicator. Based on some adequate clinical data, which for the purpose of the following discussion do not need to be made specific, we monitor its posterior distribution $p(x)$ and the risk of the clinical indicator exceeding some tolerance threshold

$$
P(X>\tau)=\int_{\tau}^{\infty} p(x) d x
$$

If this risk exceeds some critical value $\alpha, P(X>\tau)>\alpha$, medical treatment, for instance in the form of medication, is provided. While potentially only aiming at a symptomatic relief, this treatment is assumed to interfere with the patient's physiology or pathophysiology and affect the clinical disease indicator. Let $Y \in \mathbb{Y}$ denote a random variable that represents the value of the disease indicator upon medical intervention, and let $f$ describe the effect of the treatment: $y=f(x)$. This treatment effect implies a transformation of the probability distribution of the disease indicator:

$$
p_{y}(y)=\int_{-\infty}^{\infty} \delta(y-f(x)) p_{x}(x) d x
$$

where $\delta($.$) is the Dirac delta function. The consequence is a potential prediction bias:$

$$
P(Y>\tau)=\int_{\tau}^{\infty} p(y) d y \neq P(X>\tau)=\alpha
$$

which needs to be accounted for in the clinical decision process. As a simple illustration, assume the posterior distribution of the clinical indicator prior to the medical intervention is normal, $p_{x}(x)=N\left(x \mid \mu, \sigma^{2}\right)$, with mean $\mu=0$ and variance $\sigma^{2}=1$, and that the effect of the medical treatment is a shift of the clinical indicator by $\psi \in \mathbb{R}^{+}$:

$$
f(x)=\left[\begin{array}{ll}
x & \text { if } x \leq \tau \\
x-\psi & \text { if } x>\tau
\end{array}\right.
$$

This can be written in a more concise form as

$$
f(x)=[\mathcal{H}(x-\tau)](x-\psi)+[1-\mathcal{H}(x-\tau)] x
$$


where $\mathcal{H}$ is the Heaviside step function, which is equal to 1 if the argument is positive, and 0 otherwise. We obtain $p(y)$ by inserting (5) into (2) and making use of the following feature of the Dirac delta function:

$$
\delta(y-f(x))=\sum_{i} \frac{1}{\left|f^{\prime}\left(x_{i}\right)\right|} \delta\left(x-x_{i}\right)
$$

where $\left\{x_{i}\right\}$ are the roots of $y-f(x)=0$. Inserting (5) and (6) into (2) gives:

$$
p_{y}(y)=\left[\begin{array}{ll}
p_{x}(y) & \text { if } y<\tau-\psi \\
p_{x}(y)+p_{x}(y+\psi) & \text { if } \tau-\psi \leq y \leq \tau \\
p_{x}(y+\psi) & \text { if } y>\tau
\end{array}\right.
$$

The apparent probability of the disease indicator to exceed the critical threshold $\tau$ will therefore be evaluated as

$$
P(Y>\tau)=\int_{\tau}^{\infty} p_{y}(y) d y=\int_{\tau}^{\infty} p_{x}(y+\psi) d y=\int_{\tau+\psi}^{\infty} N(y \mid 0,1) d y=\bar{G}(\tau+\psi)
$$

where $\bar{G}=1-G$ and $G($.$) is the normal cumulative distribution function, whereas the actual probability is$

$$
P(X>\tau)=\int_{\tau}^{\infty} p_{x}(x) d y=\int_{\tau}^{\infty} N(y \mid 0,1) d y=\bar{G}(\tau)
$$

Since $\bar{G}($.$) is strictly monotonously decreasing, \bar{G}(\tau+\psi)<\bar{G}(\tau)$, the apparent probability is biased and systematically underestimates the risk of exceeding the critical threshold $\tau: P(Y>\tau)<P(X>\tau)$. Hence, by ignoring the effect of the treatment on the clinical indicator variable, any clinical decision support system based on this indicator variable will systematically underestimate the patient's state of risk.

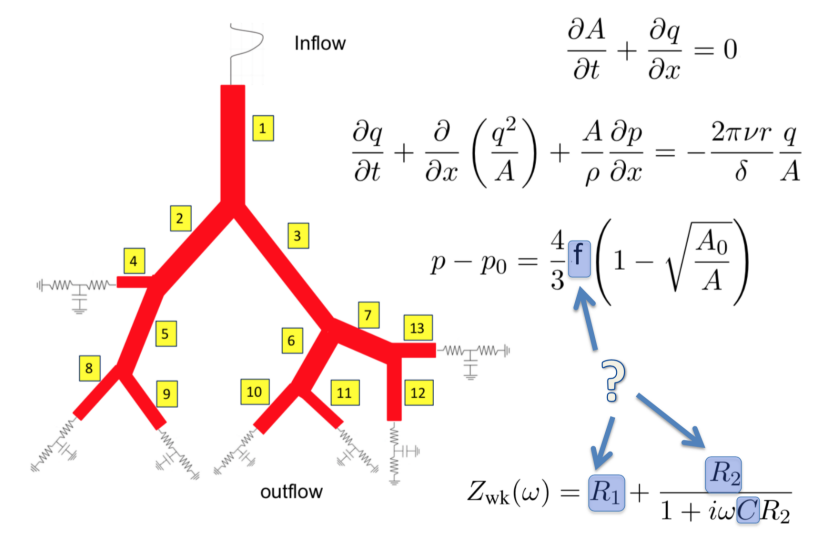

Fig. 1: Fluid dynamics model of pulmonary blood circulation. See Section 2 for details and a definition of the symbols.

\section{Physiological application: pulmonary hypertension}

Pulmonary hypertension, i.e. high blood pressure in the lungs, is a major risk factor for a variety of medical conditions, including inadequate coronary perfusion, stroke and heart failure. Pulmonary blood pressure can differ substantially from blood pressure in the rest of the body (the so-called systemic circuit) and, as opposed to the latter, can only be measured invasively. Standard techniques, which are based on right-heart catheterization, can have significant side effects, including internal bleeding and partial collapse of the lungs.

Recent advances in physiological modelling allow the pulmonary blood pressure to be predicted from the vasculature geometry and blood flow times series [1], which can be measured non-invasively with computed tomography angiography 


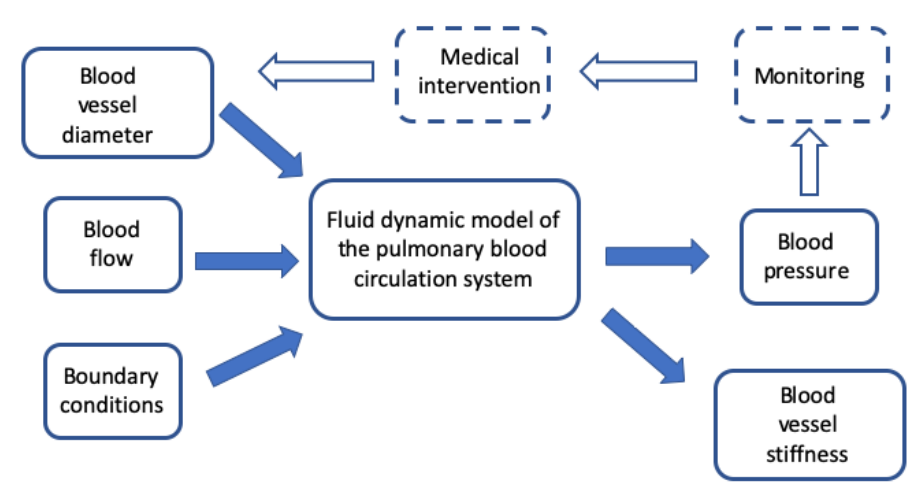

Fig. 2: Schematic representation of our physiological model of pulmonary hypertension and how it is affected by closed-loop effects following a clinical intervention. See the main text for details.

(CTA) or magnetic resonance angiography (MRA), respectively. The biophysical model depends on various boundary conditions and physiological parameters, most notably the blood vessel stiffness, which can be estimated with computational inference procedures; see [2] and [3] for details.

Figure 1 provides a graphical illustration of the model. We are given a blood vessel network, obtained e.g. from CTA or MRA. Blood flow $(q)$ and pressure $(p)$ in this network are modelled as a function of location $(x)$ and time $(t)$ with partial differential equations, which have been derived under certain physical assumptions (that the fluid is viscous, homogeneous and incompressible, and that the flow is axisymmetric, laminar and without swirls). The blood flow at the main pulmonary artery (at the top of the figure) is measured with ultrasound. The effect of the small blood vessels downstream, which are too small to be reliably extracted with CTA or MRA, are approximated with electronic circuits composed of two resistances $\left(R_{1}\right.$ and $\left.R_{2}\right)$ and one capacitance $(C)$ (so-called Windkessel elements), which determine the frequency $(\omega)$ dependent impedance $Z_{w k}(\omega)$. The model depends on various material parameters (blood density $\rho$ and viscosity $v$ ), which can be directly measured, and various geometry parameters (boundary layer thickness $\delta$, blood vessel radius $r$, cross section area $A$ and reference area $A_{0}$ ), which can be measured with CTA or MRA. However, the clinically critical parameter is the blood vessel stiffness $f$, which can not be measured in vivo and has to be inferred, along with the Windkessel parameters, from the blood flow. For details, see [1].

Figure 2 provides a schematic illustration of the way this model would be used as part of a clinical decision support system. Given the geometry of the vasculature, most notably the blood vessel diameters (measured with CTA or MRA), the blood flow (measured with ultrasound) and various boundary conditions (obtained from statistical inference, see [1]), the model allows the prediction of the pulmonary blood pressure and the blood vessel stiffness (with the statistical inference techniques described in [2] and [3]).

\section{Simulation study}

Our simulations are based on the pulmonary circulation model described in [1]. The blood vessel geometry of the larger blood vessels has been obtained from a CTA scan in a healthy mouse, the effect of the small terminal blood vessels is mimicked with Windkessel elements consisting of two resistances and a capacitance. This gives three parameters that define downstream boundary conditions of the partial differential equations (PDEs) describing the blood flow through the pulmonary circuit. We also assume that the blood flow at the main pulmonary artery (MPA) is measured (with ultrasound), which provides the upstream boundary conditions for the PDEs. Following [1] and [2], we assume the same stiffness parameter in all blood vessels, which adds one further parameter to the physiological model. We further assume that the blood flow in the two daughter vessels of the MPA are measured (with MRI). Our data used for inference are the time courses of the blood flows through three blood vessels, marked by the numbers 1-3 in Figure 1. The parameters to be inferred are the vessel stiffness and three Windkessel parameters. Once these parameters have been estimated, the blood pressure in the MPA can be predicted. 
To mimic the effect of clinical interventions, we monitor the blood pressure in the MPA, and provide an in-silico vasodilator whenever the pressure exceeds a critical threshold. Since this is a proof-of-concept study, we use data from mice rather than humans, and set as an arbitrary threshold the peak pressure found in the hypoxic mice used in the study of Qureshi et al. [1]. We simulate the effect of the vasodilator by increasing the diastolic trough diameter of all blood vessels by the same percentage amount, whose value is determined by the requirement that upon medical intervention, the peak blood pressure in the MPA must not exceed the critical value by more than 5\%. This bandwidth defines the uncertainty that remains when explicitly including the closed-loop effect caused by the medical intervention in the model. We compare that with naive parameter inference that does not include any correction for the medical intervention, and assumes the diastolic blood vessel diameter to be fixed. We quantify the effect of ignoring the feedback loop with the percentage estimation error of the vessel stiffness. The results can be found in Table 1

Table 1: Closed-loop effect and its correction in the biophysical modelling of pulmonary hypertension. Systolic blood flow can be measured with MRI; the initial geometry of the vasculature, including the diastolic diameter of the main pulmonary artery (MPA), is available from an initial CT scan. The biophysical model allows the prediction of the pulmonary systolic blood pressure (column 1) and the vessel stiffness (columns 2-3) with the statistical inference procedure described in [2] and [3]. The table shows the relative blood vessel stiffness estimation error (median and $95 \%$ posterior credible interval) without (column 2) and with a correction for the closed-loop effect that results from medical interventions triggered by model predictions. Since this is a simulation study ([1]), the true vessel stiffness is known. Computational Bayesian inference was carried out with the MCMC scheme described in [2] and [3].

\begin{tabular}{ccc}
\hline $\begin{array}{c}\text { Peak blood pressure } \\
\text { exceeding threshold }\end{array}$ & $\begin{array}{c}\text { Relative error without } \\
\text { closed-loop correction }\end{array}$ & $\begin{array}{c}\text { Relative error with } \\
\text { closed-loop correction }\end{array}$ \\
\hline $25 \%$ & $1.51 \%(1.04 \%, 1.97 \%)$ & $-2.0 \mathrm{e}-03 \%(-0.37 \%, 0.37 \%)$ \\
$50 \%$ & $2.52 \%(2.15 \%, 2.90 \%)$ & $-1.4 \mathrm{e}-03 \%(-0.47 \%, 0.46 \%)$ \\
$75 \%$ & $61.5 \%(60.5 \%, 62.4 \%)$ & $-0.26 \%(-7.78 \%, 6.49 \%)$ \\
\hline
\end{tabular}

\section{Conclusions}

In a clinical application, the prediction of high pulmonary blood pressure above a critical threshold will trigger the administration of vasodilators, whose effect is the increase of the vessel diameter. However, as illustrated in Figure 2, this causes a closed-loop effect, whereby the prediction from the model causes an action that alters the conditions under which the original prediction was obtained. In our simulation study, summarised in Table 11. we demonstrate that ignoring this closed-loop effect leads to a systematic bias in the estimation of the blood vessel stiffness, which is a critical risk indicator for vessel wall rupture, stroke and right-ventricle heart failure [4]. Allowing for the medical intervention and including the ensuing feedback loop in the statistical inference corrects this bias and leads to a substantially improved estimation of the stiffness parameter.

\section{Acknowledgments}

This work has been funded by EPSRC, grant reference numbers EP/R018634/1 (on closed-loop data science), EP/S030875/1 and EP/N014642/1 (on multiscale soft-tissue mechanics), and the Royal Society of Edinburgh, grant reference number 62335.

\section{References}

[1] M. U. Qureshi, M. J. Colebank, L. M. Paun, L. E. Fix, N. Chesler, M. A. Haider, N. A. Hill, D. Husmeier, and M. S. Olufsen, "Hemodynamic assessment of pulmonary hypertension in mice: a model-based analysis of the disease mechanism," Biomechanics and Modeling in Mechanobiology, vol. 18, pp. 219-243, 2018.

[2] L. M. Paun, M. U. Qureshi, M. Colebank, N. A. Hill, M. S. Olufsen, M. A. Haider, and D. Husmeier, "MCMC methods for inference in a mathematical model of pulmonary circulation," Statistica Neerlandica, vol. 72, pp. 306-338, 2018.

[3] L. M. Paun, M. Colebank, M. U. Qureshi, M. Olufsen, N. Hill, and D. Husmeier, "MCMC with delayed acceptance using a surrogate model with an application to cardiovascular fluid dynamics," Proceedings of the International Conference on Statistics: Theory and Applications (ICSTA 2019), no. 28, 2019.

[4] Y. Chen, F. Shen, J. Liu, and G. Yang, "Arterial stiffness and stroke: de-stiffening strategy, a therapeutic target for stroke," Stroke and Vascular Neurology, vol. 2, pp. 65-72, 2017. 